\title{
Microdosimetry Using Rhodamine B Within Macro- and Microsystems for Radiofrequency Signals Exposures of Biological Samples
}

\author{
Amani Nefzi, Lynn Carr, Claire Dalmay, Arnaud Pothier, Philippe Leveque, and Delia Arnaud-Cormos,
}

\begin{abstract}
Exposing living cells to a certain level of electromagnetic field (EMF) might induce some biological effects including temperature elevation. In this article, we studied two exposure systems at the macro and microscopic levels, allowing the study of the EMF effect on the biological samples exposed to 1.8-GHz signals. The macrosystem was an open transverse electromagnetic (TEM) cell that served as a dosimetry reference for defining limitations and optimal conditions for the temperature calibration using Rhodamine B (RhodB). The microfluidic microsystem was based on the coplanar waveguide (CPW) electrodes. Temperature measurements are carried out with a fluorooptic probe to extract specific absorption rate (SAR) values that are compared with numerical dosimetry, based on an FDTD method. After calibration, the fluorescence fits well with the temperature variation measured by the probe. To investigate dosimetry at a microscopic level, the fluorescence of the temperature-dependent dye RhodB was measured by fluorescence microscopy within the microfluidic channel or the biological cells. Results evidenced that the technique is applicable for RhodB concentrations higher than $1 \mu \mathrm{m}$ with a value of 50 $\mu \mathrm{m}$ recommended for reliable experiments. For steady detection and SAR assessments, temperature variations of a few tenths of degrees were required.
\end{abstract}

Index Terms - Exposure systems, fluorescence, microdosimetry, microelectrodes, microfluidics, radiofrequency (RF), rhodamine B (RhodB).

\section{INTRODUCTION}

$\mathbf{T}$ HE interaction of electromagnetic fields (EMF) with living entities has been the subject of a considerable amount of research in the past three decades. The studies concerned a broad panel of topics ranging, nonexhaustively, from the effects of telecommunications systems on health [1] to biomedica applications, such as hyperthermia, electropo-ration [2] nanosecond pulsed electric fields [3], as well as microfluidic [4] and lab-on-a-chip devices. To reach rigorous

Manuscript received June 17, 2019; revised September 16, 2019; accepted October 19, 2019. Date of publication November 13, 2019; date of current version March 4, 2020. This work was supported by the European Union's Horizon 2020 Research and Innovation Program under Grant 737164, by Region Limousin, the CNRS, and ANR Labex SigmaLim. (Corresponding author: Delia Arnaud-Cormos.)

A. Nefzi, L. Carr, C. Dalmay, A. Pothier, and P. Leveque are with the University of Limoges, CNRS, XLIM, UMR 7252, F-87000 Limoges, France.

D. Arnaud-Cormos is with the University of Limoges, CNRS, XLIM, UMR 7252, F-87000 Limoges, France, and also with the Institut Universitaire de France (IUF), 75005 Paris, France (e-mail: delia.arnaud-cormos@xlim.fr).

Published paper Digital Object Identifier 10.1109/TMTT.2019.2950191 conclusions, these studies require the accurate assessment of EMF parameters such as electric field, temperature, and specific absorption rate (SAR) [5]. However, due to the lack of appropriate instrumentation, assessing these exposure parameters is challenging, in particular at microscopic scales.

This article aims to investigate dosimetry at a micro-scopic level and further contribute to the characterization of exposure systems. The microdosimetry is based on the fluorescence intensity of the dye, Rhodamine B (RhodB), which is temperature-dependent. Few studies have reported RhodB temperature measurements in the context of $900-\mathrm{MHz}$ exposures [6]-[9].

Biological samples were exposed in this article to dif-ferent radiofrequency (RF) incident powers at a frequency of 1.8 $\mathrm{GHz}$ commonly used in mobile phone telecommuni-cation systems and for bioelectromagnetic investigations [1]. A first complete study was carried out in an open transverse electromagnetic (TEM) cell exposure system that contains a FluoroDish. Although TEM cells have been used as a macrosystem in bioelectromagnetic studies for in vitro experiments [1], [10], [11], real-time microscopic observations studies with FluoroDishes are rather uncommon.

In recent years, microsystems, such as thin interdigitated arrays of electrodes with micrometer gaps, have been used for the impedancemetry real-time monitoring of the cellular changes caused by the exposure to RF [12]. In such microsystems, numerical simulations evidenced a significant difference between the SAR values of the whole volume and the local SAR at the electrodes and the cellular level.

Microfluidic and lab-on-chip technologies are currently the subjects of an important research effort [13]. By reducing analyte and reagent volumes and allowing for multiplexing, they have a large range of potential applications in chemical, biomedical, or environmental fields [14]. In most lab-on-chip applications, effective control of temperature is a key factor in the optimization of the chip's performance, especially when biological samples are exposed to RF [15], [16]. Under common conditions, RF power is limited to avoid significant heating [17]. Microexposure systems can, however, provide high EMFs that can induce temperature increase not perfectly controlled. To avoid potential temperature increase, very low conductivity media that do not provide the best conditions for biological sample experiments can be used [18]. In these 


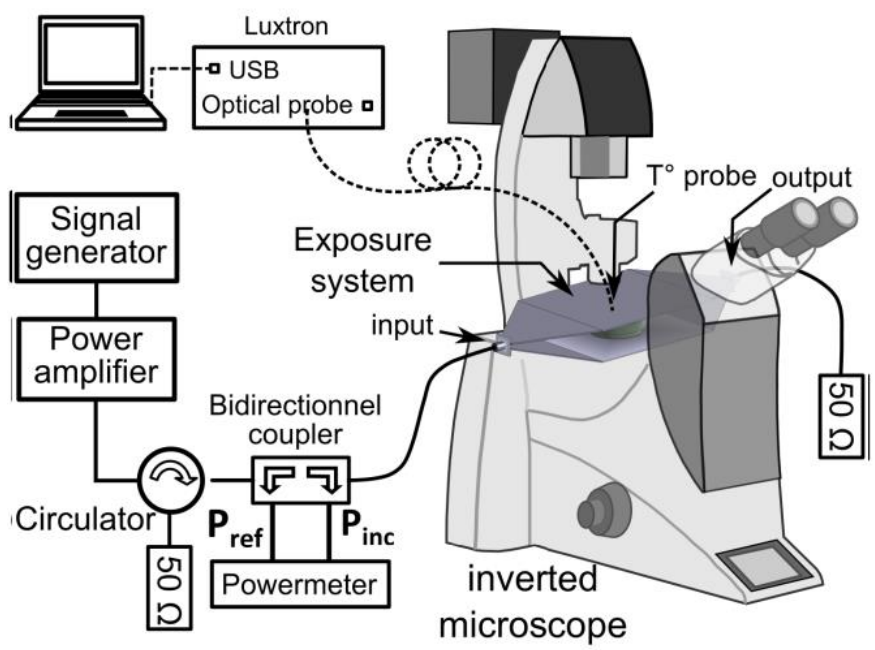

Fig. 1. Biological sample RF exposure in a TEM cell with a FluoroDish, temperature measurements setup, and real-time microscopic measurements of RhodB fluorescence.

microsystems, it is, therefore, important to characterize temperature variations accurately. Temperature measurements in a microfluidic channel, with a very limited volume of analysis that includes biological materials, require the development of specific protocols, as conventional physical probes are not suitable [19]. Although challenging, microdosimetric measure-ments using the temperature-dependent dye, RhodB, at the electrode/cell level would be highly useful in assessing local exposure parameters.

In this article, we first propose a dosimetric characterization of an exposure system based on a TEM cell that contains a FluoroDish. This exposure system served as a dosime-try reference for defining limitations and optimal conditions for the RhodB-based temperature calibration. A microfluidic microsystem was specifically designed for RhodB fluorescence measurements. As an intermediate step, a glass slide, similar to the microfluidic system support, holding a sample drop was exposed and characterized in the TEM cell.

This article is organized as follows. Section II presents the exposure systems, the setup and exposure protocols, and the numerical modeling and RhodB fluorescence measurements. Section III details the results in terms of SAR values and distri-butions, RhodB fluorescence, and temperature measurements. Conclusions are given in Section IV.

\section{Materials AND Methods}

\section{A. RF Exposure Setup}

The setup for exposures to RF at $1.8 \mathrm{GHz}$ of biological samples is shown in Fig. 1. It is composed of a continuous-wave (CW) RF signal generator (HP8648B, Hewlett-Packard, USA) connected to a 44-dB gain power amplifier (M.19.40.50, Nuclétudes, France). The amplifier is connected to a bidirectional coupler that monitors incident and reflected powers at the exposure system input. Depending on the exposure system (e.g., TEM cell), a 50- high-power load can be connected a)
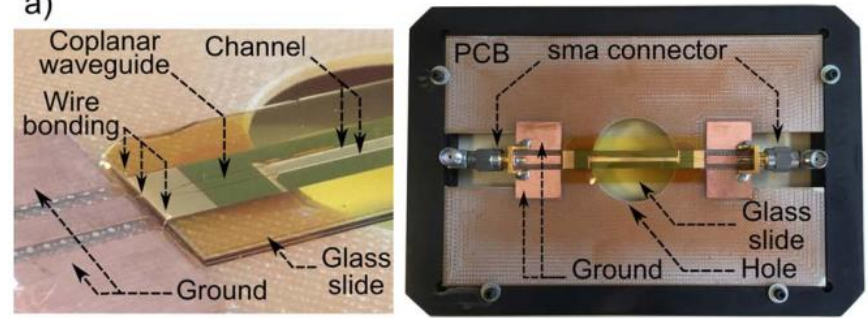

b)

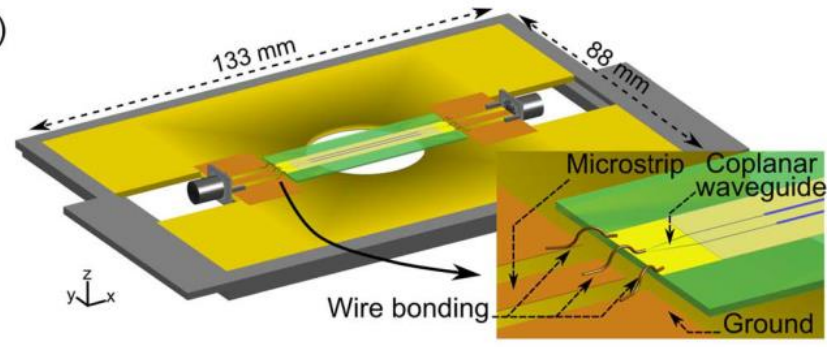

c)
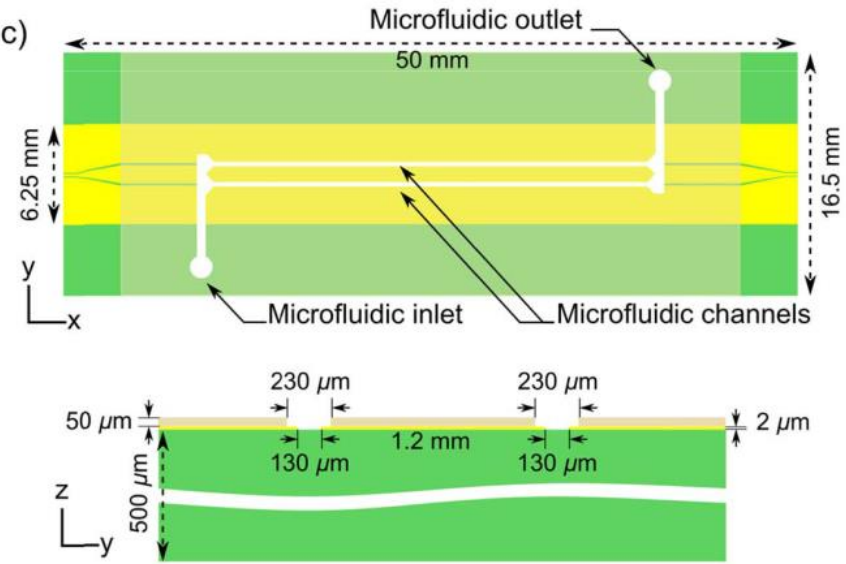

Fig. 2. Microsystem design and dimensions. (a) Photographs of the microfluidic microsystem based on CPW electrodes and of the microsys-tem mounted on a PCB designed to be inserted on the microscope stage.

(b) 3-D view of the global system with electrical connections (numerical model extracted from the FDTD software). (c) Schematic top view and sectional view. The center CPW line is narrow at the beginning to allow conventional on-wafer probing with ACP-40-A-GSG Cascade probes connected to a vector network analyzer.

to the output port to absorb the transmitted power and match the impedance.

\section{B. Macroexposure System}

To calibrate the RhodB-based temperature technique, exper-iments on biological samples exposed to RF were first con-ducted with an open TEM cell exposure system [20], [21] (Fig. 1). The TEM cell contains a FluoroDish (World Precision Instruments \#FD35-100) as a biological holder. A 20-mm aperture on the TEM lower plate allows real-time microscopic fluorescence measurements.

\section{Microexposure System}

Fig. 2 shows the microsystem designed for microflu-idic applications and for microdosimetric characterization in 
response to RF fields in the microwave range that was investigated with the proposed RhodB technique.

The microelectrodes for the microdosimetry experiments are based on a coplanar waveguide (CPW) designed to expose the fluid injected in the microfluidic channel. The microelectrodes were designed to ensure impedance matching of the CPW when the microchannel is filled with a biological sample, usually an aqueous saline-based solution presenting a relative permittivity of 76 and an electrical conductivity equal to $2.3 \mathrm{~S} / \mathrm{m}$ at $1.8 \mathrm{GHz}$ and room temperature.

The developed microsystem is based on $2-\mu \mathrm{m}$ gold electrodes evaporated and patterned by standard photolithography on a 500- $\mu \mathrm{m}$-thick fused silica substrate. The microfluidic channels were defined using a negative photosensitive polymer, named DF 1050. This polymer was laminated on the chip to form a 50- $\mu \mathrm{m}$-high microchannel network [See Fig. 2(c)]. First, experiments were led in static conditions with no flow in the channel. Hence, the fluid was injected in the microchannels using a micropipette and channels were closed with a cover glass slide to prevent evaporation during exposure and monitoring of the fluorescent level.

To adapt the microsystem with the real-time monitoring of the RhodB fluorescence setup on the microscope stage (Fig. 1), a printed circuit board (PCB) was designed allowing applying the RF signal through SMA connectors. Fig. 2(a) shows the system composed of the microelectrodes connected to the PCB that was dimensioned to be inserted on the microscope stage. Copper lines adapted at 50 have been designed to ensure the connections between the CPW and the SMA connectors through wire-bonding [See Fig. 2(b)].

\section{Numerical Modeling and Simulations}

1) TEM Cell Containing a FluoroDish: The numerical dosimetry was performed with a 3-D electromagnetic software package based on the FDTD method. The TEM and the FluoroDish, with the biological sample, were modeled, as shown in Fig. 3. Perfect electric conductors were assigned to model the metallic plates of the TEM cell. The 50-impedance ports were used for the input feed source and the output load. The FluoroDish glass and the plastic permittivities were set to 7.5 and 2.5, respectively. The biological buffer solution containing the RhodB was modeled with the following dielectric properties at $1.8 \mathrm{GHz}$ : conductivity of $2.3 \mathrm{~S} / \mathrm{m}$, relative permittivity of 76 , and mass density of $1000 \mathrm{~kg} / \mathrm{m}^{3}$. These properties were measured at room temperature using a dielectric probe immersed in the buffer solution $(85070 \mathrm{E}$ Dielectric Probe, Agilent, USA). The simulations were performed at a frequency of $1.8 \mathrm{GHz}$. During the experiments, the temperature increases for a few degrees, inducing a modification of the liquid's dielectric properties of less than 5\% that can also be reported on the SAR. The TEM structure was meshed with a nonuniform mesh size. For increased accuracy, the biological sample and the FluoroDish glass bottom were meshed with a grid mesh of $100 \mu \mathrm{m}$. The top of the microscope objective is also considered in the simulations as a perfect conductor with a lens modeled as a glass with a relative permittivity of 7.5.
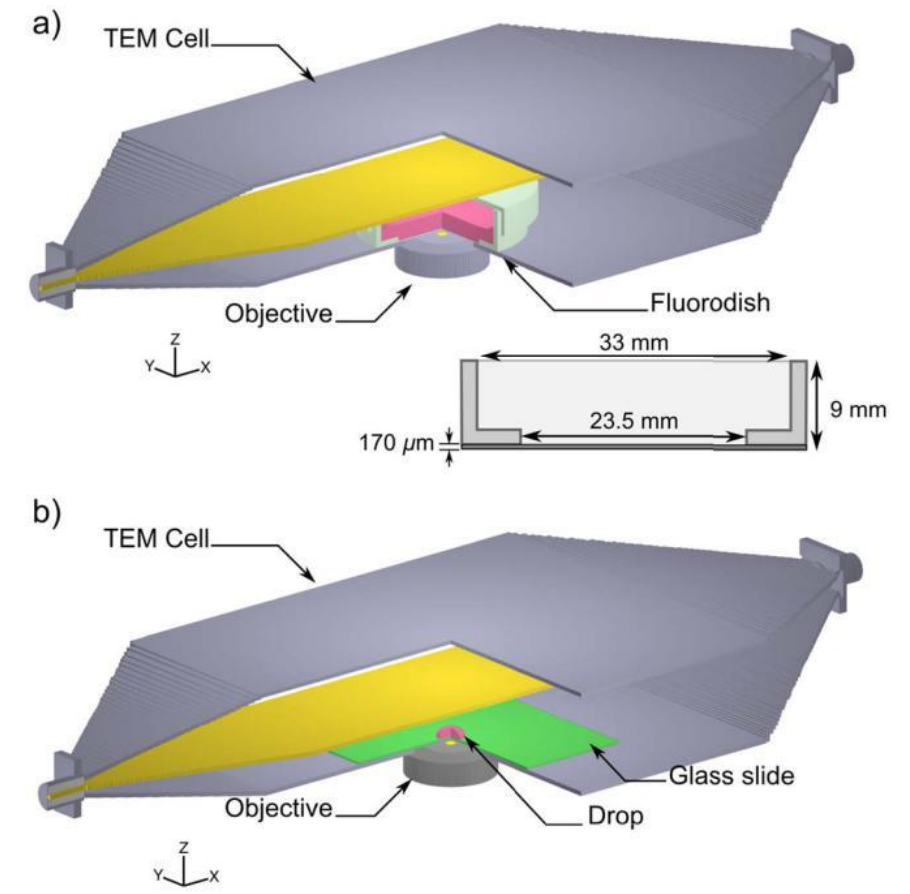

Fig. 3. TEM cell with (a) FluoroDish that contains the biological sample (numerical model extracted from the FDTD software, FluoroDish dimensions) and (b) glass slide with a drop of biological sample.

2) TEM Cell Containing a Glass Slide With a Drop of Biological Sample: The TEM cell containing a glass slide with a drop of biological medium was also modeled and simulated. The relative permittivity of the glass slide was 3.8. Its dimensions were $50 \mathrm{~mm} \times 50 \mathrm{~mm} \times 0.5 \mathrm{~mm}$. A half ellipse with a radius of $3 \mathrm{~mm}$ and a height of $2 \mathrm{~mm}$ was implemented to model the small drop corresponding to the $35-\mu \mathrm{L}$ volume of the biological sample. All other geometric, dielectric, and simulation parameters were set as for the modeling of the TEM cell containing the FluoroDish.

3) Microfluidic Microsystem: The designed microsystem illustrated in Fig. 2 was modeled, as shown in Fig. 2(b). The $2-\mu \mathrm{m}$-thick gold electrodes, the metallic parts of the PCB that ensured the connections with the SMA connectors, and the wire bonding were considered as perfect electric conductors. The relative permittivities of the $500-\mu \mathrm{m}$-thick fused silica substrate, the $50-\mu \mathrm{m}$ polymer, and the PCB substrate were 3.8 , 3.58, and 4.3. Compared with the losses induced in the liquid, the losses of the DF 1050 and other related material are small and were often neglected in the simulations. A nonuniform meshing was implemented with a mesh size of $5 \mu \mathrm{m}$ for the simulations of the CPW microelectrodes.

\section{E. Exposure Protocol}

Temperature variation following exposure to $1.8-\mathrm{GHz} \mathrm{RF}$ at different incident powers was measured by optical fiber probes. The RhodB fluorescence variation was also measured in parallel. For reference and calibration purposes, two optical fiber temperature probes (Luxtron One, Lumasense Technologies, CA, USA) were used. The probes were placed in the center of the FluoroDish containing the biological solution 
and outside the exposure system, respectively. Images for measuring fluorescence intensity were collected using two microscope objectives: a $63 \times$ or a $20 \times$ objective.

\section{F. Rhodamine B Fluorescence Measurements and Analysis}

RhodB fluorescence was either measured in solution, by adding $3 \mathrm{~mL}$ of RhodB with different concentrations in HEPES-buffered salt solution (HBSS) to a FluoroDish, or in RhodB loaded cells. The 50- $\mu \mathrm{m}$ RhodB concentration was used for experiments except for the study of RhodB concentration influence where $10,1,0.1$, and $0.01 \mu \mathrm{m}$ were explored. RhodB (excitation: $553 \mathrm{~nm}$, emission: $627 \mathrm{~nm}$ ) was observed by epifluorescence using a solid-state light engine (Spectra 7, Lumencor), coupled to the microscope (DMI6000, Leica) by a 1-mm quartz fiber. Emitted light was separated by excitation with a dichroic beam- splitter (89100BS, Chroma) and selected using emission filters controlled by a filter wheel (MAC 6000, Ludl). Images were captured using an electronmultiplying charge-coupled device camera (EMCCD Evolve 512 , Roper) with $512 \times 512$ pixels. Images were collected every 1 or $15 \mathrm{~s}$ depending on the experiments. ImageJ was used for image analysis. For cell-free experiments, fluorescence intensity was measured using a region of interest that covered the whole imaged area. For experiments using cells, which were adherent to the glass bottom of the FluoroDish, images were background-corrected and fluorescence intensity was measured in the regions of interest manually drawn around cells.

\section{G. Biological Sample Labeling}

For experiments with cells, the human glioblastoma cell line U-87 MG (brain cancer cells) was used. Cells were grown in complete culture medium (MEM supplemented with $10 \%$ fetal bovine serum, glutamine, 100 units $/ \mathrm{mL}$ penicillin, and 100 $\mu \mathrm{g} / \mathrm{mL}$ streptomycin; Invitrogen) in an incubator at $37^{\circ} \mathrm{C}$ and $5 \% \mathrm{CO} 2$. Cells were subcultured onto plastic FluoroDishes (WPI) at a density of 1-2 $10^{6}$ cells $/ \mathrm{mL}$ and allowed to adhere and grow for $48 \mathrm{~h}$ prior to experiments. Before imaging, cells were loaded with RhodB (Sigma) using a slightly modified protocol from [9] by incubating with $50-\mu \mathrm{m}$ RhodB in HBSS $(\mathrm{NaCl} 121 \mathrm{mM}, \mathrm{KCl}$

$5.4 \mathrm{mM}, \mathrm{MgCl}_{2} 0.8 \mathrm{mM}, \mathrm{NaHCO} 36 \mathrm{mM}$, D-glucose $5.5 \mathrm{mM}$, HEPES $25 \mathrm{mM}, \mathrm{CaCl} 21.8 \mathrm{mM}, \mathrm{pH} 7.3$ ) for $1 \mathrm{~h}$ at $37^{\circ} \mathrm{C}$. Following this incubation, cells were washed with HBSS to remove excess unloaded dye. A volume of $3 \mathrm{~mL}$ of HBSS was used for imaging.

\section{H. Dosimetric Assessments}

Along with the electric field and temperature, the SAR is a commonly determined parameter to assess the exposure of biological samples to RF. The SAR was extracted from measurements and simulations using the following equations:

$$
\begin{aligned}
\text { SAR } & C \frac{\partial T}{}(\mathrm{~W} / \mathrm{kg}) \\
= & \partial t t=t_{0}
\end{aligned}
$$

where $C$ is the specific heat capacity of the biological sample,

$4186 \mathrm{~J} /(\mathrm{kg} \cdot \mathrm{K})$ and $\partial \mathrm{T} / \partial \mathrm{t}$ is the initial slope of the temperature
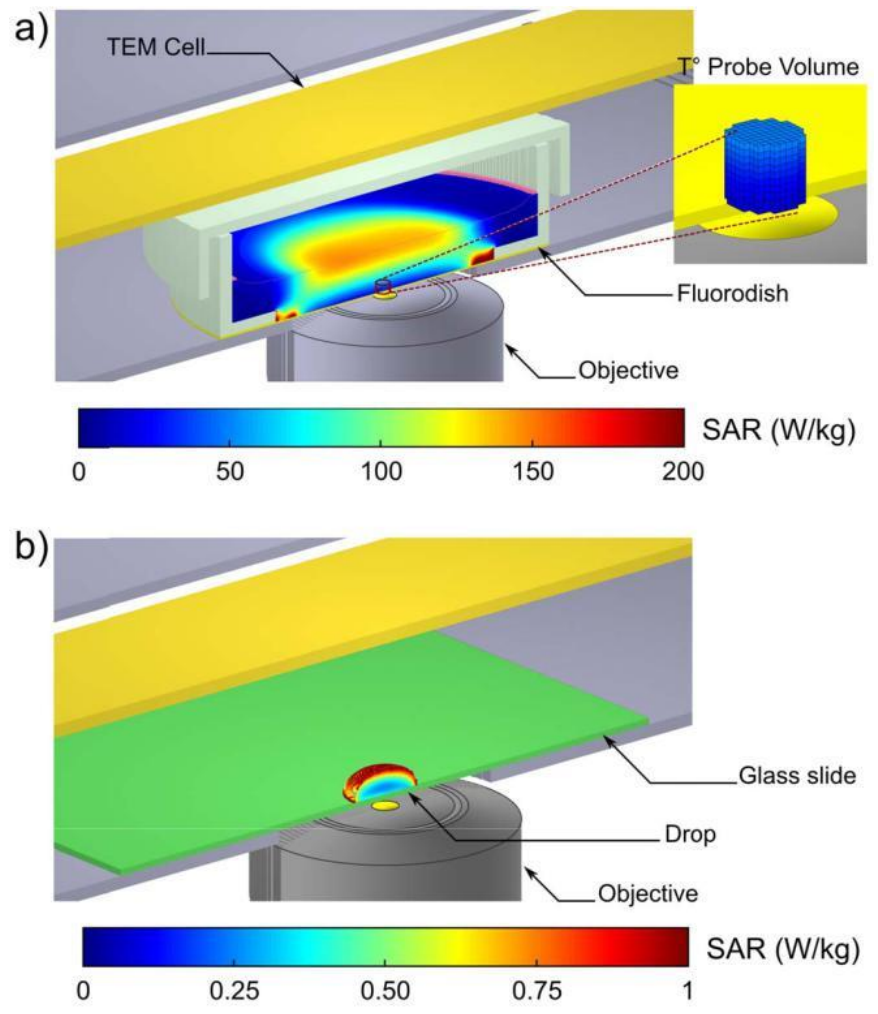

Fig. 4. SAR spatial distribution within the biological sample inside (a) FluoroDish placed in the TEM cell. Inset: Probe volume 1-mm cylinder for comparisons with Luxtron probe measured SAR. (b) Drop placed on a glass slide inside the TEM cell. SAR values are normalized per 1-W incident power for an objective at a working distance of $200 \mu \mathrm{m}$.

increase versus time and

$$
\mathrm{SAR}=\frac{\sigma E_{2}}{2 \rho}(\mathrm{W} / \mathrm{kg})
$$

where $E$ is the electric field amplitude $(\mathrm{V} / \mathrm{m}), \rho$ is the mass density $\left(\mathrm{kg} / \mathrm{m}^{3}\right)$, and $\sigma$ is the electrical conductivity $(\mathrm{S} / \mathrm{m})$ of the biological sample.

\section{RESUlTS}

\section{A. Dosimetry of the TEM Cell Containing a FluoroDish and a Glass Slide With a Biological Sample Drop}

Fig. 4 shows the numerical simulations results in terms of SAR distribution (a transversal cut along the center of the FluoroDish and the glass slides are shown). The distance corresponding to the gap between the bottom of the biological holder and the top of the microscope objective is defined as the working distance. All SAR values are normalized per 1-W incident power. For the FluoroDish [See Fig. 4(a)], the SAR value averaged over the whole biological solution volume is $41.7 \pm 43.8 \mathrm{~W} / \mathrm{kg}$ with a $200-\mu \mathrm{m}$ working distance. The computed SAR value averaged over a cylinder of 1-mm diameter and height corresponding to the Luxtron probe measurement volume is defined as the probe volume. The probe volume $\mathrm{SAR}$ is equal to $43.1 \pm 10.1 \mathrm{~W} / \mathrm{kg}$. The microfluidic support is formed from a glass slide. To model this system, a glass slide containing a drop of the biological sample, which was covered to limit evaporation, was first studied under exposure within 
a)

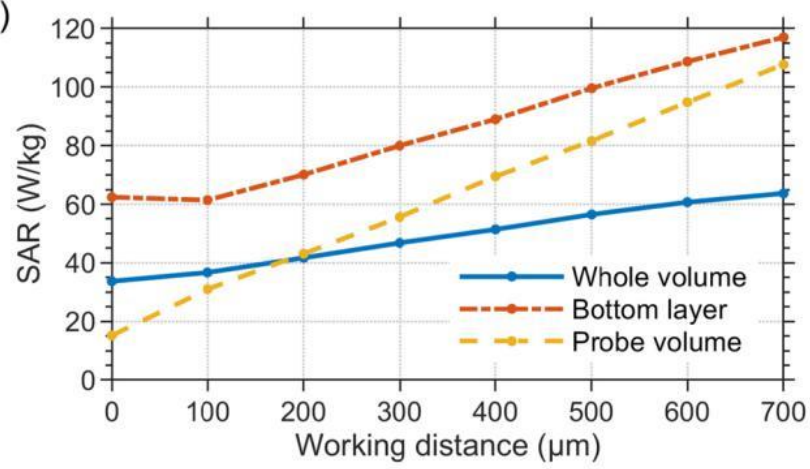

b)

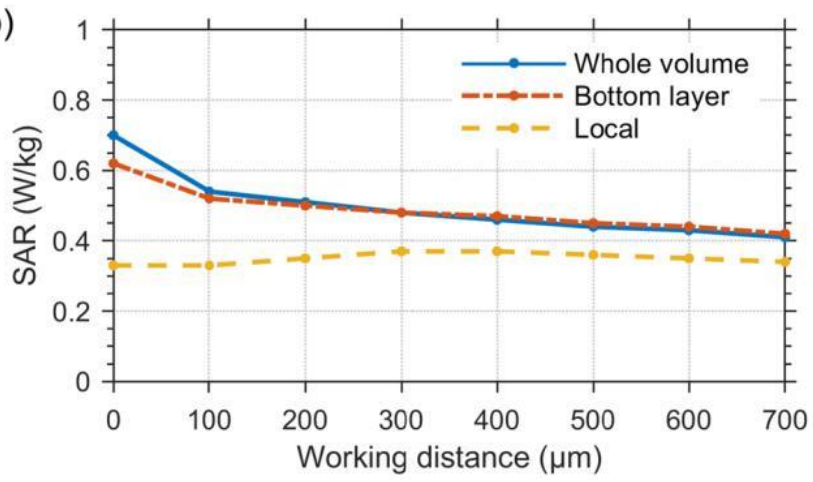

Fig. 5. Variation of SAR values (whole volume, bottom layer at the cell level, and temperature probe volume) versus the microscope objective position (the working distance corresponds to the gap between the bottom of the biological holder and the top of the objective). SAR values are normalized per 1-W incident power. (a) Within the biological sample inside the FluoroDish placed in the TEM cell. (b) Within a drop placed on a glass slide inside the TEM cell.

the TEM cell. This setup served as a calibration for further measurements with the microfluidic system. For the glass slide containing the drop [See Fig. 4(b)], the SAR value averaged over the whole drop volume is $0.51 \pm 0.15 \mathrm{~W} / \mathrm{kg}$ with a $200-\mu \mathrm{m}$ working distance. The SAR at the bottom layer of the drop, averaged over a slice of $100-\mu \mathrm{m}$ height corresponding to the simulation mesh size, is equal to $0.36 \pm 0.02 \mathrm{~W} / \mathrm{kg}$. The inhomogeneous SAR distribution within the whole volume is not critical as we conduct temperature measurements and fluorescence acquisitions over small volumes and thin imaging slices. In the fluorescence measurement area, the SAR values on a horizontal plane are rather homogenous. Considering a height of $1 \mathrm{~mm}$, the probe volume SAR standard deviation remains within acceptable values lower than $25 \%$.

Fig. 5 shows SAR values obtained from numerical simulations (whole volume, bottom layer at the cells level, probe volume) versus the working distance. The probe volume corresponds to the simulation SAR over the equivalent Luxtron probe volume located at the probe tip [See Fig. 4(a)]. For the FluoroDish [See Fig. 5(a)], the SAR values depend on the microscope objective position. The interference of the objective on the SAR values can be explained by the coupling between the objective and the hole of the TEM cell. We have considered reducing the hole; however, this would limit the use of the $63 \times$ objective, which requires to be placed in contact with the biological holder. Thus, the objective working distance must be considered for an
TABLE I

Localized (Luxtron Probe) and Simulated (Probe Volume) SAR Values at $1.8 \mathrm{GHz}$. SAR Values ARe Normalized PER 1-W INCIDENT Power

\begin{tabular}{ccc}
\hline \hline \multirow{2}{*}{ Objective (distance $\mu \mathrm{m}$ ) } & \multicolumn{2}{c}{$\mathrm{SAR}(\mathrm{W} / \mathrm{kg})$} \\
\cline { 2 - 3 } & Measurements & Simulation \\
\hline Objective 63x (0) & $13.9 \pm 2.0$ & $11.8 \pm 4.6$ \\
Objective 20x (200) & $45.4 \pm 2.8$ & $43.1 \pm 10.1$ \\
Objective 20x (400) & $64.1 \pm 4.3$ & $68.5 \pm 14.8$ \\
\hline \hline
\end{tabular}

accurate dosimetry. In Fig. 5(b), local SAR values are obtained from the simulated SAR over the volume corresponding to $100-\mu \mathrm{m}$-high (spatial resolution), 1-mmwide slice. This vol-ume is related to the fluorescence signal measurements. For the glass slide containing the drop [See Fig. 5(b)], the SAR values slightly decrease and they are less sensitive to the microscope objective.

Table I shows the SAR values obtained from simulations and measurements for two microscope objectives $63 \times$ and $20 \times$ placed in contact, at 200 and $400 \mu \mathrm{m}$, respectively. In simulations, the standard deviation is computed from the voxels in the selected volume of the SAR, as illustrated in the inset of Fig. 4(a). In the experiments, the standard deviation is due to different temperature acquisitions and from the SAR estimation using temperature measurements (2).

Results show good agreement between simulations and measurements for all configurations.

\section{B. Optimizations of the RhodB-Based Dosimetric Technique}

RhodB fluorescence measurements at the level of biological cells or thin imaging slices are a rather complex process. We observed that the control of the room temperature is important. For instance, the air-conditioning can cause $\pm 1^{\circ} \mathrm{C}$ in room temperature. In addition, high temperature varia-tions can modify the focus of the microscope. The setup of the RhodB measurement technique thus requires simul-taneous fine-tuning and optimization of different parameters related to the RF exposure system, the imaging fluorescence acquisitions, and the biological sample preparation. Different parameters were studied with the TEM cell containing the FluoroDish such as the influence of the incident power, the concentration of RhodB, and the duration of RF exposures. Moreover, the relation between the \% change in fluorescence and temperature rise is dependent on several factors. In our experiments, no significant variations depending, for example, on the $\mathrm{pH}$ of the culture medium were observed [9], [19].

1) Influence of the Incident Power: Different measurements were carried out to study the sensitivity of the RhodB to the incident power. For these experiments, we used $3 \mathrm{~mL}$ of HBSS containing $50-\mu \mathrm{m}$ RhodB in a FluoroDish. The FluoroDish was placed inside the TEM cell, and measurements at the different incident powers $(10,5,1$, and $0.5 \mathrm{~W})$ were carried out with exposure durations (RFon) varying from $30 \mathrm{~s}$ to $10 \mathrm{~min}$.

To extract the temperature, a conversion coefficient $\alpha$ was defined as the ratio between temperature variation and normalized fluorescence variation, i.e., the ratio between the 

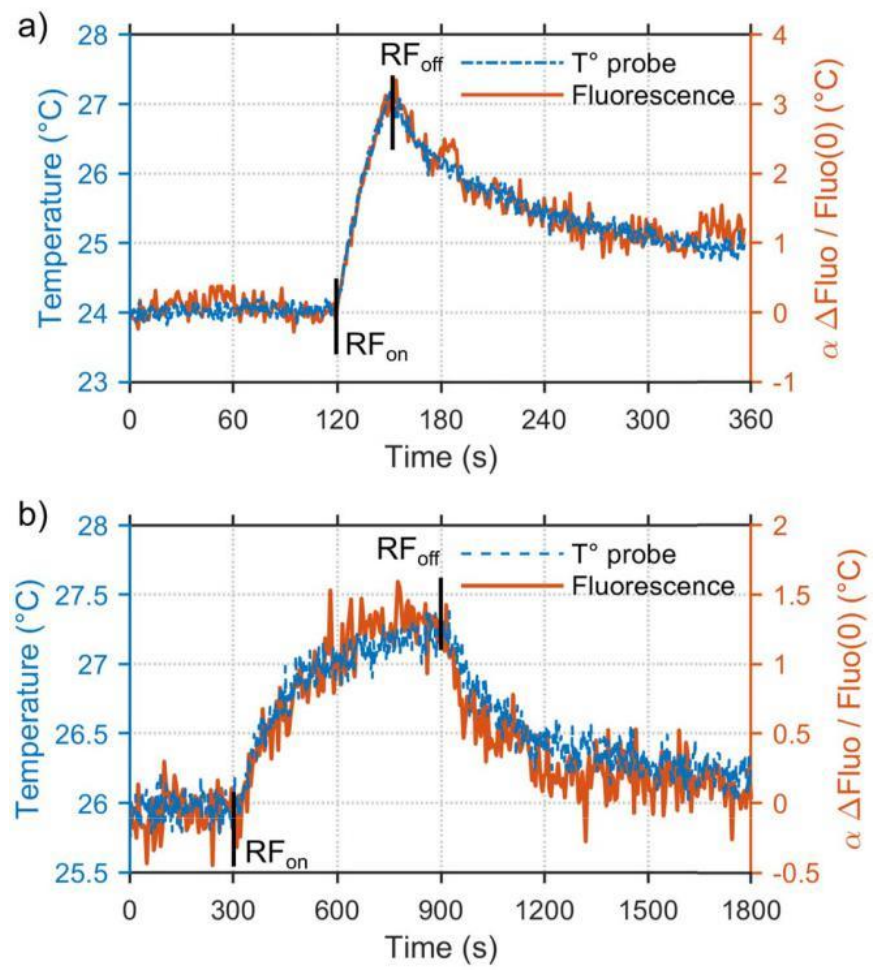

Fig. 6. Experimental results of temperature obtained with the optical fiber probe and extracted from Rhod B fluorescence measurements. The biological samples, $3 \mathrm{~mL}$ of HBSS with $50-\mu \mathrm{m}$ RhodB, were exposed to $1.8 \mathrm{GHz}$. Fluorescence measurements were performed with the $20 \times$ objective. (a) Data at incident power of $10 \mathrm{~W}$ for an RF exposure duration of $30 \mathrm{~s}$. (b) Data at an incident power of $0.5 \mathrm{~W}$ for an RF exposure duration of $10 \mathrm{~min}$.

fluorescence values and the initial fluorescence before RFon

$$
\alpha=-\frac{T^{\circ}}{\text { Fluo/Fluo }(0)}(\circ \mathrm{C} / \mathrm{u}) .
$$

The normalized fluorescence variation was slightly less than $2 \% 1^{\circ} \mathrm{C}$, and the conversion coefficient $\alpha$ was assessed at $57 \pm 2.5$ (mean $\pm \mathrm{SD})$.

Typical results presented in Fig. 6 show a temperature elevation of $3^{\circ} \mathrm{C}$ and $1.5^{\circ} \mathrm{C}$, for $10-\mathrm{W} 30$-s RFon and $0.5-\mathrm{W}$ 10-min RFon, respectively. Plots evidence good agreement between the temperature and fluorescence values even for low incident powers $(0.5 \mathrm{~W})$.

2) Influence of the RhodB Concentration: To study the influence of RhodB concentration on its fluorescence, measurements were performed with concentrations of 50,10, 1 , 0.1 , and $0.01 \mu \mathrm{m}$. The incident power was varied from 0.5 to $10 \mathrm{~W}$, and the same protocol of $10 \mathrm{~min}$ or $30 \mathrm{~s}$ of RFon was applied. The results reveal a good concordance between the temperature and the RhodB fluorescence for concentrations higher than $1 \mu \mathrm{m}$ for a significant increase of temperature (typically $1{ }^{\circ} \mathrm{C}$ ). These experiments point out the dependence of the RhodB fluorescence to its bulk concentration.

Table II shows the SAR values obtained from measurements at 1.8 GHz for different incident power and RhodB concen-trations. As observed, the SAR values normalized per 1-W incident power extracted from all fluorescence measurements present a high level of consistency.
TABLE II

SAR VAlues at $1.8 \mathrm{GHz}$ For DifFerent InCident Powers AND RhodB Concentrations. SAR Values Are Normalized PeR 1-W Incident Power

\begin{tabular}{ccccc}
\hline \hline & \multicolumn{4}{c}{ SAR (W/kg) } \\
\cline { 2 - 5 } Concentration & $0.5 \mathrm{~W}$ & $1 \mathrm{~W}$ & $5 \mathrm{~W}$ & $10 \mathrm{~W}$ \\
\hline $1 \mu \mathrm{M}$ & - & $63.3+4.6$ & $66.8 \pm 5.5$ & $63.5+3.7$ \\
$10 \mu \mathrm{M}$ & $54.0 \pm 7.2$ & $64.5 \pm 2.1$ & $65.5 \pm 6.4$ & $61.8+5.5$ \\
$50 \mu \mathrm{M}$ & $61.8 \pm 2.3$ & $66.8 \pm 4.0$ & $66.8 \pm 5.5$ & $65.8 \pm 7.7$ \\
\hline \hline
\end{tabular}

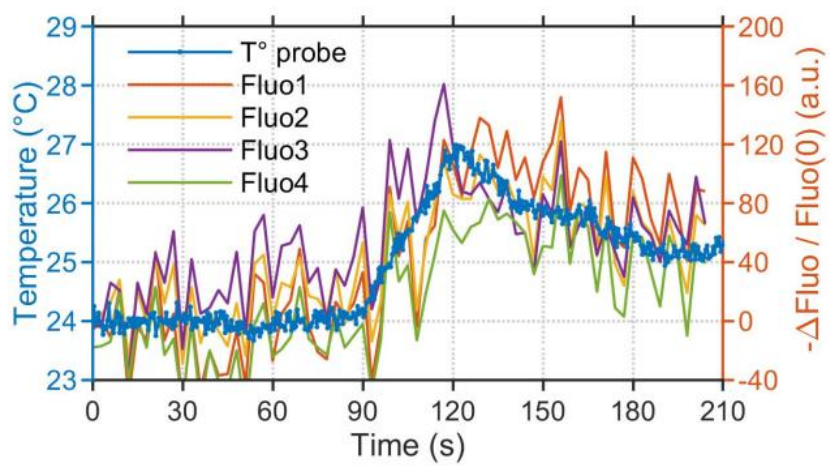

Fig. 7. Experimental results of temperature versus Rhod B fluorescence variation obtained with the $20 \times$ objective at a working distance of $200 \mu \mathrm{m}$ for four different U-87 MG clusters of 4-5 cells. The cells were exposed to 1.8 $\mathrm{GHz}$, at $10-\mathrm{W}$ incident power, for a duration of $30 \mathrm{~s}$, in the FluoroDish placed in the TEM cell.

The results of this macrodosimetry are reproducible, and this measurement technique thus calibrated and validated can inform us about the temperature variation of a sample at the microscopic scale.

Fig. 7 shows the experimental results of temperature versus Rhod B fluorescence variation obtained for clusters of few U$87 \mathrm{MG}$ adherent cells. The cells are exposed to $1.8 \mathrm{GHz}$ at $10-$ $\mathrm{W}$ incident power, for durations of $30 \mathrm{~s}$, in the FluoroDish placed in the TEM cell. Results evidence the fluorescence variation that can be correlated with the temperature variation. It should be noted that for the fluorescence acquisitions, only the area of each cell is considered. This can explain the noise on the fluorescence measurements. As the cell clusters differ, it is necessary to normalize the fluorescence-level reference. Indeed, within the different clusters, there are regions without fluorescence. As the mean value of the fluorescence is different from one cluster to another, a normalization was applied to merge all the clusters. Therefore, the calibration factor cannot be applied.

\section{Dosimetry of the Microfluidic Microsystem}

\section{1) Electromagnetic Frequency Characterization: Fig. 8} shows S-parameters that characterize the transmitted $\left(S_{21}\right)$ and reflected $(S 11)$ powers by the microfluidic system. Sparameters were obtained from measurements achieved by conventional on wafer probing with ACP-40-A-GSG Cascade probes connected to a calibrated Rohde \& Schwarz ZVA-24 network analyzer. The microfluidic channel is either empty or filled with the biological sample, i.e., a drop of HBSS. Fig. 8(a) shows that the results of the empty or filled 

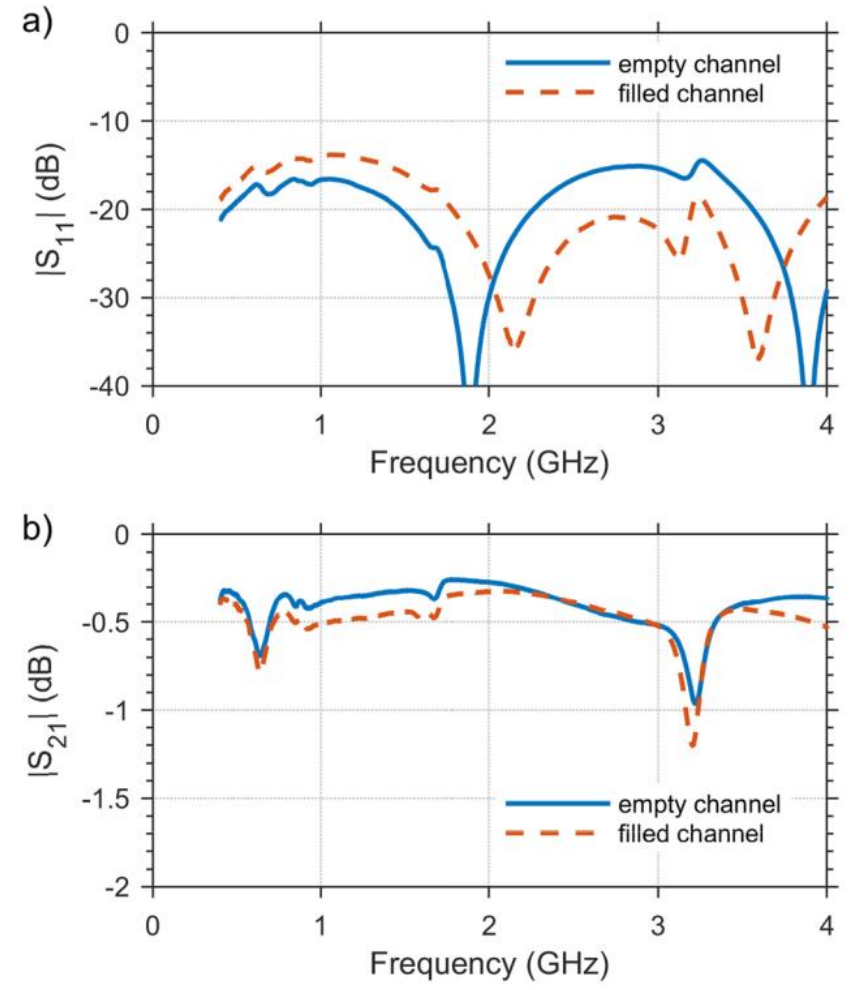

Fig. 8. S-Parameters over a wide frequency range of the microfluidic microsystem; the microfluidic channel is empty or filled with the biological sample, i.e., a drop of HBSS. (a) Reflection (S11) coefficient. (b) Transmission (S21) coefficient.

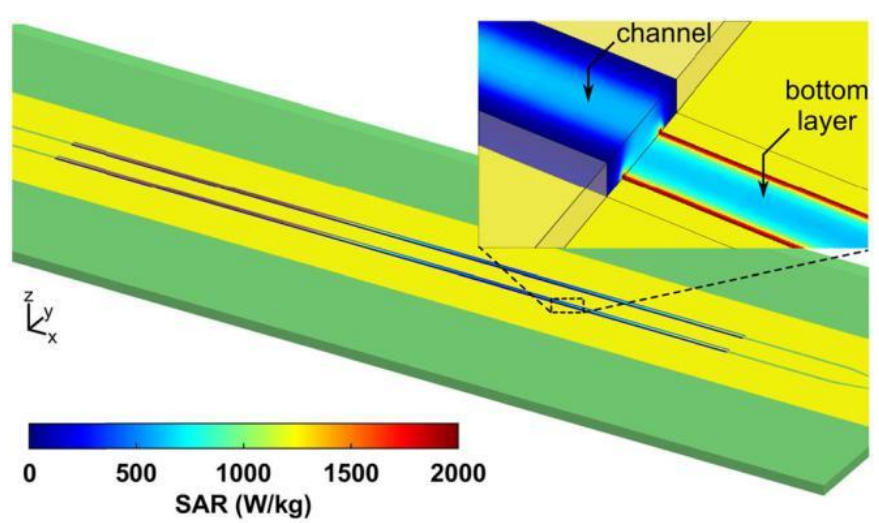

Fig. 9. SAR spatial distribution within the microfluidic channel filled a drop of HBSS covered by a glass slide. SAR values are normalized per 1-mW incident power.

microfluidic channel slightly differ particularly in terms of $\left|S_{11}\right|$ frequency position minima that can be related to the resonance frequency of the microsystem. For both configurations, however, the reflection coefficient $\left|S_{11}\right|$ remains lower than $-10 \mathrm{~dB}$ for the considered frequency band, ensuring a good impedance matching. Fig. 8(b) shows that the transmission $\left(S_{21}\right)$ coefficients for both configurations are higher than $-1 \mathrm{~dB}$ for frequencies up to $3 \mathrm{GHz}$. These measurements validated the relevance of using such a device for the exposure of biological cells, since the energy will be effectively transferred to the biological medium inside the microfluidic channel.

Fig. 9 shows the SAR spatial distribution within the microfluidic channel filled with a drop of HBSS covered by

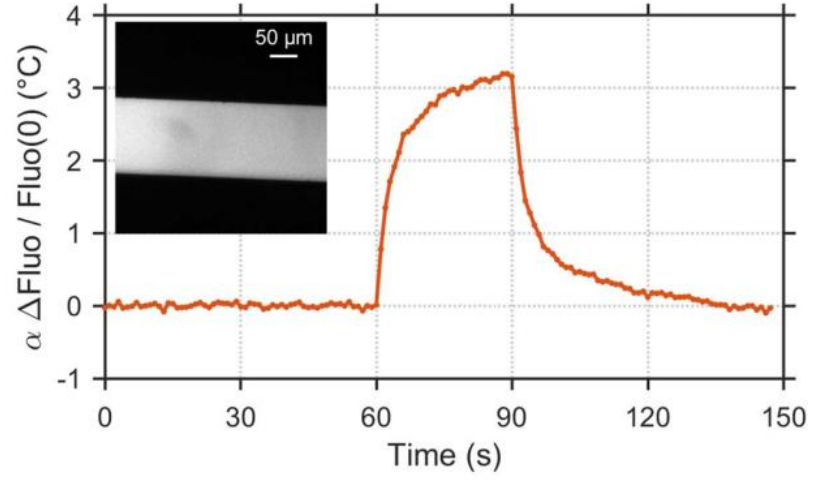

Fig. 10. RhodB fluorescence variation obtained with the $20 \times$ objective within the microfluidic channel containing a drop of HBSS with $100-\mu \mathrm{m}$ RhodB. The microsystem is exposed to $1.8 \mathrm{GHz}$ at $0.2-\mathrm{W}$ incident power for a duration of $30 \mathrm{~s}$.

a glass slide. SAR values are normalized per 1 -mW incident power. A decrease in the SAR values can be observed along the channel. The inset in Fig. 9 highlights the SAR distribution along a fragment of the channel and only at the electrode level on the right-hand side. The SAR values are rather homogenous along the channel thickness except on the electrodes and the channel edges.

2) RhodB Fluorescence Measurements: The RhodB fluorescence variation measured within the microfluidic chan-nel is shown in Fig. 10. The microsystem is exposed to $1.8 \mathrm{GHz}$ at $0.2-\mathrm{W}$ incident power during $30 \mathrm{~s}$. For these measurements, the drop of HBSS that fills the channel was labeled with 100$\mu \mathrm{m}$ RhodB to enhance fluorescence emis-sion. The fluorescence result is related to the RF exposure with a fast variation during the 30-s RF delivery. An SAR value of 14.5 $\mathrm{kW} / \mathrm{kg}$ was extracted from the initial slope of the fluorescence/temperature. Indeed, the fluorescence signal measurements depend on the sensitive volume. For small microchannels, very high SAR values and, therefore, high tem-perature variation differences between the simulations and the measurements may exist. Two phenomena can be evidenced. First, the initial slope of the temperature may be very steep and SAR determination from this slope can be limited by the camera temporal resolution $(1 \mathrm{~s}$ in our experiments). Second, as the involved volume is very small, the temperature variation and dispersion are significant. In this context, the calibration for small channels may require tuning of the fluorescence-sensitive volume and comparisons with simulated SAR. For macroscopic systems (FluoroDish and drop), the sensitive volume is not critical as the SAR is rather homogenous over the 1-mm bottom layer.

\section{CONCLUSION}

In this article, a temperature dosimetric technique based on RhodB fluorescence variation was set up and optimized. The technique serves to complete numerical dosimetry results for $\mathrm{RF}$ exposure systems. The exposure setup was based on a FluoroDish, containing $3 \mathrm{~mL}$ of HBSS, inserted into the TEM cell. The dosimetry assessment was carried out both numerically, with the FDTD method to obtain SAR 
distribution values, and experimentally, using a Luxtron probe and RhodB for temperature measurement.

For the setup and optimization of the RhodB-based dosi-metric technique, different parameters such as RhodB con-centration and the incident power directly related to the SAR thresholds were studied. From the results, it can be evidenced that the technique is applicable for RhodB concentrations higher than 1 $\mu \mathrm{m}$ with a value of $50 \mu \mathrm{m}$ recommended for reliable experiments. As for the measurements with the optical fiber temperature probe, a temperature variation of a few tenths of degrees is required for detection and SAR assessment. One experiment with cells adherent to a FluoroDish exposed in a TEM cell illustrating that fluorescence variation can be correlated with the temperature variation was reported.

This technique is highly relevant for dosimetric characterizations at the microscopic level and suitable for the microexposure system assessment of local exposure parameters. In this article, a microfluidic microsystem was specifically designed for RF exposures and RhodB fluorescence measurements.

\section{REFERENCES}

[1] A. Paffi et al., "Considerations for developing an RF exposure system: A review for in vitro biological experiments," IEEE Trans. Microw. Theory Techn., vol. 58, no. 10, pp. 2702-2714, Oct. 2010.

[2] M. L. Yarmush, A. Golberg, G. Serša, T. Kotnik, and D. Miklav̌cǐc, "Electroporation-based technologies for medicine: Principles, applications, and challenges," Annu. Rev. Biomed. Eng., vol. 16, pp. 295-320, Jul. 2014.

[3] K. H. Schoenbach et al., "Ultrashort electrical pulses open a new gateway into biological cells," Proc. IEEE, vol. 92, no. 7, pp. 1122-1137, Jul. 2004.

[4] C. Dalmay et al., "A microfluidic biochip for the nanoporation of living cells," Biosensors Bioelectron., vol. 26, no. 12, pp. 4649-4655, Aug. 2011.

[5] The International Commission on Non-Ionizing Radiation Protection, "Guidelines for limiting exposure to time-varying electric, magnetic, and electromagnetic fields (up to $300 \mathrm{GHz}$ )," Health Phys., vol. 74, no. 4, pp. 494-522, Apr. 1998.

[6] J. F. Bermingham, Y. Y. Chen, R. L. McIntosh, and A. W. Wood, "A measurement and modeling study of temperature in living and fixed tissue during and after radiofrequency exposure," Bioelectromagnetics, vol. 35, no. 3, pp. 181-191, Apr. 2014.

[7] S. Kohler, R. P. O'Connor, T. D. T. Vu, P. Leveque, and D. ArnaudCormos, "Experimental microdosimetry techniques for bio-logical cells exposed to nanosecond pulsed electric fields using microfluorimetry," IEEE Trans. Microw. Theory Techn., vol. 61, no. 5, pp. 2015-2022, May 2013.

[8] Y. Y. Chen and A. W. Wood, "Application of a temperature-dependent fluorescent dye (Rhodamine B) to the measurement of radiofrequency radiation-induced temperature changes in biological samples," Bioelectromagnetics, vol. 30, no. 7, pp. 583-590, Oct. 2009.

[9] D. Moreau, C. Lefort, R. Burke, P. Leveque, and R. P. O'Connor, "Rhodamine B as an optical thermometer in cells focally exposed to infrared laser light or nanosecond pulsed electric fields," Biomed. Opt. Express, vol. 6, no. 10, pp. 4105-4117, Oct. 2015.

[10] S. Kohler, T. D. T. Vu, P. T. Vernier, P. Leveque, and D. ArnaudCormos, "Characterization of a TEM cell-based setup for the exposure of biological cell suspensions to high-intensity nanosecond pulsed electric fields (nsPEFs)," in IEEE MTT-S Int. Microw. Symp. Dig., Montreal, QC, Canada, Jun. 2012, pp. 1-3.

[11] C. Iftode and S. Miclaus, "Design and validation of a TEM cell used for radiofrequency dosimetric studies," Prog. Electromagn. Res., vol. 132, pp. 369-388, Oct. 2012.

[12] M. A. García-Fernández et al., "Dosimetric characteristics of an EMF delivery system based on a real-time impedance measurement device," IEEE Trans. Biomed. Eng., vol. 63, no. 11, pp. 2317-2325, Nov. 2016.

[13] E. Verpoorte and N. F. De Rooij, "Microfluidics meets MEMS," Proc. IEEE, vol. 91, no. 6, pp. 930-953, Jun. 2003.
[14] G. M. Whitesides, "The origins and the future of microfluidics," Nature, vol. 442, no. 27, pp. 368-373, Jul. 2006.

[15] Y. Inoue, R. Obara, M. Nakano, and J. Suehiro, "Concentration of bacteria in high conductive medium using negative dielectrophoresis," in Proc. IEEE Int. Conf. Ind. Technol., Seville, Spain, Mar. 2015, pp. 3336-3340.

[16] R. Manczak et al., "Tracking cancer cells with microfluidic high frequency DEP cytometer implemented on BiCMOS lab-on-chip platform," in IEEE MTT-S Int. Microw. Symp. Dig., Philadelphia, PA, USA, Jun. 2018, pp. 104-107.

[17] H. Li et al., "Distributed effect in high-frequency electroporation of biological cells," IEEE Trans. Microw. Theory Techn., vol. 65, no. 9, pp. 3503-3511, Sep. 2017.

[18] J. Leroy et al., "Microfluidic biosensors for microwave dielectric spectroscopy," Sens. Actuators A, Phys., vol. 229, pp. 172-181, Jun. 2015.

[19] J. J. Shah, M. Gaitan, and J. Geist, "Generalized temperature measurement equations for rhodamine B dye solution and its application to microfluidics," Anal. Chem., vol. 81, no. 19, pp. 8260-8263, Oct. 2009.

[20] M. Soueid et al., "Delivery devices for exposure of biological cells to nanosecond pulsed electric fields," Med. Biol. Eng. Comput., vol. 56, no. 1, pp. 85-97, Jan. 2018.

[21] M. Soueid et al., "Electromagnetic analysis of an aperture modified TEM cell including an ITO layer for real-time observation of biolog-ical cells exposed to microwaves," Prog. Electromagn. Res., vol. 149, pp. 193-204, Nov. 2014.

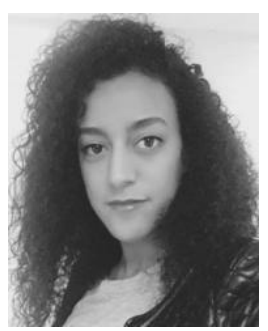

Amani Nefzi was born in Tunis, Tunisia, in 1993. She received the master's degree in electronics from the University of Limoges, Limoges, France, in 2017, where she is currently pursuing the Ph.D. degree in electronics engineering at National Center of Scientific Research (CNRS), XLIM Research Institute.

Her current research interests include the development of electromagnetic and nanosecond pulsed electric fields exposure systems for the study of the health and therapeutic effects.

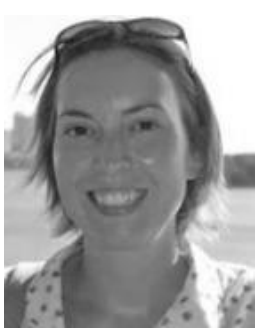

Lynn Carr received the B.Sc. degree (Hons) in biochemistry from University College London, London, U.K., and the master's degree in biotechnology and the Ph.D. degree from the University of Limoges, Limoges, France.

As part of the XLIM Research Institute's BioEM Group, her interests focus on the effects of nanosecond pulsed electric fields on cancer cells with a particular focus on cell signaling.

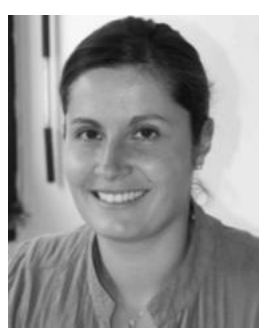

Claire Dalmay was born in Limoges, France, in 1983. She received the Ph.D. degree in electri-cal engineering from the University of Limoges, Limoges, in 2009.

She holds a post-doctoral position with the C.N.R.S., SATIE-BIOMIS, IFR d'Alembert, ENS Cachan, Cachan, France, focusing on the develop-ment of microfluidic biochips for cell electroporation and cell handling. She is currently an Assistant Professor with the XLIM Laboratory, University of Limoges/C.N.R.S. Her current research interests

include the development of microsystems for biomedical applications and additive microfabrication technologies for RF and microwave components. 


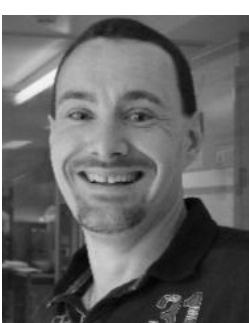

Arnaud Pothier (M'10) received the Ph.D. degree in electrical engineering from the University of Limoges, Limoges, France, in 2003.

$\mathrm{He}$ is currently a Full-Time Senior Researcher with the XLIM Research Institute. His current research interest includes RF biosensors for labelfree cell analysis.

Dr. Pothier is a member of the EuMA Society and the Technical Program Committees of IMS, EuMW, and IMBIOC Conferences and previously served as the Chair for the IEEE MTT-S Technical Committee MTT-10 on Biological Effect and Medical Applications.

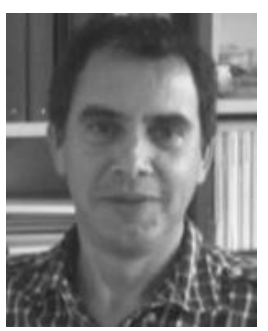

Philippe Leveque (M'03) was born in Poitiers, France, in 1964. He received the Ph.D. degree from the University of Limoges, Limoges, France, in 1994.

In 1995, he joined C.N.R.S. He is involved in the development of dosimetry and exposure setups for health-risk assessment in cooperation with biological and medical research groups. He is currently a Senior Scientist with CNRS and the Group Leader of Bioelectromagnetics Team with the XLIM Research Institute focusing on nanopulse application. Since

2018, he has been a member of the International Bioelectrics Consortium. His current research interest includes the scattering problems of electromagnetic waves, particularly in the time domain.

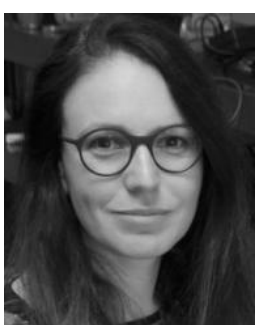

Delia Arnaud-Cormos (M'05) was born in Cugir, Romania, in 1978. She received the Diplôme d'Ingénieur degree from the Institute of Com-puter Science and Communication (IFSIC), Rennes, France, in 2002, and the master's and Ph.D. degrees from the National Institute of Applied Sciences (INSA), Rennes, in 2003 and 2006, respectively.

Since 2007, she has been with the Bioelectromagnetics Team, XLIM Institute, University of Limoges/C.N.R.S., Limoges, France, as an Associate Professor. In 2012, she joined the Pulsed

Power Group, Department of Electrical Engineering, University of Southern California (USC), Los Angeles, SC, USA, where she developed research with the Biological Applications Research Team. Since 2018, she has been a Junior Member with the Institut Universitaire de France (IUF), Paris, France, and a member of the International Bioelectrics Consortium. Her current research interests include nanosecond pulses/microwave exposure system setup and dosimetric characterization for bioelectromagnetic studies. 